PoS $\quad \begin{aligned} & \text { PROCEEDINGS } \\ & \text { OF SCIENCE }\end{aligned}$

\title{
Review and interpretation of new heavy states discovered at the $B$ factories
}

\section{Marina Nielsen*}

Instituto de Física, Universidade de São Paulo, C.P. 66318, 05389-970 São Paulo, SP, Brazil

E-mail: mnielsen@if.usp.br

I discuss some recent discoveries in the spectroscopy of the charmonium-like resonances, and some of their theoretical interpretations are reviewed.

8th Conference Quark Confinement and the Hadron Spectrum September 1-6, 2008

Mainz. Germany

\footnotetext{
* Speaker.
} 


\section{Introduction}

The study of spectroscopy and the decay properties of the heavy flavor mesonic states provides us useful information about the dynamics of quarks and gluons at the hadronic scale. The remarkable progress at the experimental side, with various high energy machines, like the $B$-factories, has opened up new challenges in the theoretical understanding of heavy flavor hadrons.

The $B$-factories, the PEPII at SLAC in the U.S.A., and the KEKB at KEK in Japan, were constructed to test the Standard Model mechanism for CP violation. They are $e^{+} e^{-}$colliders operating at a $\mathrm{CM}$ energy near $10,580 \mathrm{MeV}$. The $B \bar{B}$ pairs produced are measured by the $\mathrm{BaBar}$ (SLAC) and Belle (KEK) collaborations.

Although the main goal of the $B$-factories was the observation of $\mathrm{CP}$ violation, their contribution to the area of charmonium spectroscopy have been by far more important. At the quark level, the $b$ quark decays weakly to a $c$ quark accompained by the emission of a virtual $W^{-}$boson. Approximately half of the time, the $W^{-}$boson materializes as a $s \bar{c}$ pair. Therefore, half of the $B$ meson decays result in a final state that contains a $c \bar{c}$ pair. When these $c \bar{c}$ pairs are produced close to each other in phase space, they can coalesce to form a $c \bar{c}$ charmonium meson.

The simplest charmonium producing $B$ meson decay is: $B \rightarrow K(c \bar{c})$. Another interesting form to produce charmonium in $B$-factories is directly form the $e^{+} e^{-}$collision, when the initial state $e^{+}$or $e^{-}$occasionally radiates a high energy $\gamma$-ray, and the $e^{+} e^{-}$subsequently annihilate at a corresponding reduced CM energy. When the energy of the radiated $\gamma$-ray $\left(\gamma_{I S R}\right)$ is between 4000 and $5000 \mathrm{MeV}$, the $e^{+} e^{-}$annhilation occurs at $\mathrm{CM}$ energies that correspond to the range of mass of the charmonium mesons. Thus, the initial state radiation (ISR) process can directly produce charmonium states with $J^{P C}=1^{--}$.

In the next sections I discuss the experimental data and the possible interpretations for the recently observed $X, Y$ and $Z$ mesons.

\section{The $X(3872)$ meson}

In August 2003, Belle reported evidence for a new narrow state in the decay $B^{+} \rightarrow X(3872) K^{+} \rightarrow$ $J / \psi \pi^{+} \pi^{-} K^{+}$[1], which has been confirmed by CDF, D0 and BaBar [2]. The current world average mass is

$$
M_{X}=(3871.4 \pm 0.6) \mathrm{MeV},
$$

and its total width is less than 2.3 MeV. Belle's [3] and BaBar's [4] observation of the decay $X(3872) \rightarrow J / \psi \gamma$ determines $C=+$, opposite to the charge-conjugation of the leading charmonium candidates. Angular correlations among the final state particles from $X(3872) \rightarrow J / \psi \pi^{+} \pi^{-}$ decay strongly suggests $J^{P C}=1^{++}$quantum numbers [5].

From constituent quark models [6] the masses of the possible charmonium states with $J^{P C}=$ $1^{++}$quantum numbers are; $2{ }^{3} P_{1}(3990)$ and $3{ }^{3} P_{1}(4290)$, which are much bigger than the observed mass.

Evidence for the decay $X(3872) \rightarrow J / \psi \pi^{+} \pi^{-} \pi^{0}$ at a rate comparable to that $X(3872) \rightarrow$ $J / \psi \pi^{+} \pi^{-}$was also observed by Belle [3]:

$$
\frac{X \rightarrow J / \psi \pi^{+} \pi^{-} \pi^{0}}{X \rightarrow J / \psi \pi^{+} \pi^{-}}=1.0 \pm 0.4 \pm 0.3
$$


This observation establishes strong isospin and $\mathrm{G}$ parity violation, which is incompatible with a $c \bar{c}$ structure for $X(3872)$.

The observation of these two decays, plus the coincidence between the $X$ mass and the $D^{* 0} D^{0}$ threshold: $M\left(D^{* 0} D^{0}\right)=(3871.81 \pm 0.36) \mathrm{MeV}$ [7], inspired the proposal that the $X(3872)$ could be a molecular $\left(D^{* 0} \bar{D}^{0}+\bar{D}^{* 0} D^{0}\right)$ bound state with small binding energy [8, 9]. As a matter of fact, Tornqvist, using a meson potential model [10], essentially predicted the $X(3872)$ in 1994, since he found that there should be molecules near the $D^{*} D$ threshold in the $J^{P C}=0^{-+}$and $1^{++}$channels. The only other molecular state that is predicted in the potential model updated by Swanson is a $0^{++} D^{*} \bar{D}^{*}$ molecule at $4013 \mathrm{MeV}$ [9]. The $D^{* 0} \bar{D}^{0}$ molecule is not an isospin eigenstate and the rate in Eq.(2.2) is explained in a very natural way in this model.

Recently Belle [11] and BaBar [12] Collaborations reported a near threshold enhancement in the $D^{0} \bar{D}^{0} \pi^{0}$ system. The peak mass values for the two observations are in good agreement with each other: $(3875.2 \pm 1.9) \mathrm{MeV}$ for Belle and $(3875.1 \pm 1.2) \mathrm{MeV}$ for BaBar, and are higher than in the mass of the $X(3872)$ observed in the $J / \psi \pi^{+} \pi^{-}$channel by $(3.8 \pm 1.1) \mathrm{MeV}$. Since this peak lies about $3 \mathrm{MeV}$ above the $D^{* 0} \bar{D}^{0}$ threshold, it is very ackward to treat it as a $D^{* 0} \bar{D}^{0}$ bound state. According to Braaten [13], the peak observed in the $B \rightarrow K D^{0} \bar{D}^{0} \pi^{0}$ decay channel is a combination of a resonance below the $D^{* 0} \bar{D}^{0}$ threshold from the $B \rightarrow K J / \psi \pi^{+} \pi^{-}$decay and a threshold enhancement above the $D^{* 0} \bar{D}^{0}$ threshold. However, in an updated study [14], the new value for the mass of the near threshold enhancement in the $D^{0} \bar{D}^{0} \pi^{0}$ system reported by the Belle Collaboration is $\left(3872.6_{-0.4}^{+0.5} \pm 0.4\right) \mathrm{MeV}$, in a very good agreement with the current world average mass for the $X(3872)$ in the $J / \psi \pi^{+} \pi^{-}$mode in Eq. (2.1).

Maiani it et al. [15] advocate a tetraquark explanation for the $X(3872)$. They have considered diquark-antidiquark states with $J^{P C}=1^{++}$and symmetric spin distribution:

$$
X_{q}=[c q]_{S=1}[\bar{c} \bar{q}]_{S=0}+[c q]_{S=0}[\bar{c} \bar{q}]_{S=1} .
$$

Physical states could be expected to fall in isospin multiplets with $I=0,1$ :

$$
X(I=0)=\frac{X_{u}+X_{d}}{\sqrt{2}}, \quad X(I=1)=\frac{X_{u}-X_{d}}{\sqrt{2}} .
$$

However, due to the charm quark mass scale, annihilation diagrams are suppressed and, therefore, states are closer to mass eigenstates and are no longer isospin eigenstates. The most general states are:

$$
X_{l}=\cos \theta X_{u}+\sin \theta X_{d}, \quad X_{h}=\cos \theta X_{d}-\sin \theta X_{u},
$$

and both can decay into $2 \pi$ and $3 \pi$. Imposing the rate in Eq.(2.2), they get $\theta \sim 20^{\circ}$. They also argue that if $X_{l}$ dominates $B^{+}$decays, then $X_{h}$ dominates the $B^{0}$ decays and vice-versa. Therefore, the $X$ particle in $B^{+}$and $B^{0}$ decays are different with $[15,16]$

$$
M\left(X_{h}\right)-M\left(X_{l}\right)=(8 \pm 3) \mathrm{MeV} .
$$

There are two reports from Belle [17] and Babar [18] Collaborations for the observation of the $B^{0} \rightarrow K^{0} X$ decay. However, these reports are not consistent with each other. While Belle measures [17]:

$$
\frac{B^{0} \rightarrow X K^{0}}{B^{+} \rightarrow X K^{+}}=0.94 \pm 0.24 \pm 0.10
$$


and

$$
M(X)_{B^{+}}-M(X)_{B^{0}}=(0.22 \pm 0.90 \pm 0.27) \mathrm{MeV},
$$

BaBar measures [18]:

$$
\frac{B^{0} \rightarrow X K^{0}}{B^{+} \rightarrow X K^{+}}=0.41 \pm 0.24 \pm 0.05
$$

and

$$
M(X)_{B^{+}}-M(X)_{B^{0}}=(2.7 \pm 1.6) \mathrm{MeV} .
$$

In any case, the mass difference measurements are much larger than the prediciton in Eq.(2.6). It is interesting to notice that, using the same tetraquark structure as in ref. [15], a QCD sum rule calculation for the mass difference in Eq.(2.6) has obtained [19]:

$$
M\left(X_{h}\right)-M\left(X_{l}\right)=(3.3 \pm 0.7) \mathrm{MeV},
$$

in agreement with BaBar measurement. The same calculation [19] has obtained

$$
M_{X}=(3.92 \pm 0.13) \mathrm{GeV},
$$

while a QCD sum rule for the $X(3872)$ resonance considering it as a $\left(D^{* 0} \bar{D}^{0}+\bar{D}^{* 0} D^{0}\right)$ molecular state [20] has obtained

$$
M_{X}=(3.87 \pm 0.07) \mathrm{GeV},
$$

in a better agreement with the experimental mass. Therefore, from a QCDSR point of view, the $X(3872)$ is better described as a $D^{*} D$ molecular state than as a diquark-antidiquark state.

To summarize, there is an emerging consensus that the $X(3872)$ is a multiquark state. In favor of the tetraquark configuration is the existence of two different states decaying from $B^{ \pm}$or $B^{0}$. Therefore, it is very important the confirmation of the existence of these two states. In favor of the molecular configuration is the proximity of the $X(3872)$ mass and the $D^{*} D$ threshold.

\section{The $Y\left(J^{P C}=1^{--}\right)$family}

The $Y(4260)$ was the first one in the family observed by BaBar Collaboration [21] in the reaction

$$
e^{+} e^{-} \rightarrow \gamma_{I S R} J / \psi \pi^{+} \pi^{-},
$$

with mass $M=(4259 \pm 10) \mathrm{MeV}$ and width $\Gamma=(88 \pm 24) \mathrm{MeV}$. It was confirmed by CLEO and Belle Collaborations [22]. The $\pi \pi$ mass distribution reported in [21] peaks near $1 \mathrm{GeV}$ and this information was interpreted as consistent with the $f_{0}(980)$ decay. In a updated report [23], BaBar has confirmed the observation of the $Y(4260)$ with a mass and width

$$
M_{Y}=(4252 \pm 7) \mathrm{MeV}, \quad \Gamma_{Y}=(105 \pm 20) \mathrm{MeV} .
$$

However, the new $\pi \pi$ mass distribution shows a more complex structure.

BaBar [24] also found a broad peak in the reaction

$$
e^{+} e^{-} \rightarrow \gamma_{I S R} \psi^{\prime} \pi^{+} \pi^{-}
$$


which was confirmed by Belle [25]. Belle found that the $\psi^{\prime} \pi^{+} \pi^{-}$enhancement observed by BaBar was, in fact, produced by two distinct peaks with masses and widths:

$$
\begin{array}{lll}
Y(4360): & M=(4361 \pm 13) \mathrm{MeV}, & \Gamma=(74 \pm 18) \mathrm{MeV}, \\
Y(4660): & M=(4664 \pm 12) \mathrm{MeV}, & \Gamma=(48 \pm 15) \mathrm{MeV} .
\end{array}
$$

The masses and widths of these three states are not consistent with any of the established $1^{--}$charmonium states [26], and they can also be candidates for multiquark states or charmonium hybrids [27]. An attractive interpretation is that the $Y(4260)$ is a charmonium hybrid. Hybrids are hadrons in which the gluonic degree of freedom has been excited. The nature of this gluonic excitation is not well understood, and has been described by various models. The spectrum of charmonium hybrids has been calculated using lattice gauge theory [28]. Their result for the mass is approximately $4200 \mathrm{MeV}$, which is consistent with flux tube model predictions [29]. However, more recent lattice simulations predict that the lightest charmonium hybrid is about $4400 \mathrm{MeV}$ [30] , which is closer to the mass of the $Y(4360)$.

A critical information for understanding the structure of these states is wether the pion pair comes from a resonance state. From the di-pion invariant mass spectra shown in ref. [31] there is some indication that only the $Y(4660)$ has a well defined intermediate state consistent with $f_{0}(980)$ [31]. Due to this fact and the proximity of the mass of the $\psi^{\prime}-f_{0}(980)$ system with the mass of the $Y(4660)$ state, in ref. [32], the $Y(4660)$ was considered as a $f_{0}(980) \psi^{\prime}$ bound state. The $Y(4660)$ was also suggested to be a baryonium state [33] and a canonical $5{ }^{3} \mathrm{~S}_{1} c \bar{c}$ state [34].

In the case of $Y(4260)$, in ref. [35] it was considered as a $s c$-scalar-diquark $\bar{s} \bar{c}$-scalar-antidiquark in a $P$-wave state. Maiani et al. [35] tried different ways to determine the orbital term and they arrived at $M=(4330 \pm 70) \mathrm{MeV}$, which is more consistent with $Y(4360)$. However, from the $\pi \pi$ mass distribution in ref. [31], none of these two states, $Y(4260)$ and $Y(4360)$ has a decay with a intermediate state consistent with $f_{0}(980)$ and, therefore, it is not clear that they should have an $s \bar{s}$ pair in their structure. Also, in ref. [36], using a relativistic diquark-antidiquark picture, it was shown that the $Y(4260)$ can not be interpreted as a $\left([s c]_{S=0}[\bar{s} \bar{c}]_{S=0}\right)$ state in a $P$-wave.

If one looks at the threshold of the mesonic systems: $M\left(D(1865) \bar{D}_{1}(2420)\right) \sim 4285 \mathrm{MeV}$ and $M\left(D_{0}(2310) \bar{D}^{*}(2007)\right) \sim 4320 \mathrm{MeV}$, which have $J^{P C}=1^{--}$in $S$-wave, one sees that a molecular interpretation is also possible for $Y(4260)$ and $Y(4360)$. In refs. [37, 38] a QCD sum rule calculation for these molecular states was considered. The obtained mass for the $D_{0} \bar{D}^{*}$ state was: $m_{D_{0} \bar{D}^{*}}=(4.27 \pm 0.10) \mathrm{GeV}$ in good agreement with the $Y(4260)$ mass. In the case of the $D \bar{D}_{1}$ molecular state, the obtained mass was: $m_{D \bar{D}_{1}}=(4.19 \pm 0.22) \mathrm{GeV}$. Therefore, considering the errors and the width of the $Y(4260)$ meson, the molecular $D \bar{D}_{1}$ assignement is also possible, in agreement with the findings of ref. [39], where a meson exchange model was used to study the $Y(4260)$ meson.

The authors of ref. [37] also considered diquark-antidiquark states with $J^{P C}=1^{--}$and symmetric spin distribution:

$$
Y_{q}=[c q]_{S=1}[\bar{c} \bar{q}]_{S=0}+[c q]_{S=0}[\bar{c} \bar{q}]_{S=1},
$$

with $q$ standing for a light or a strange quark. The obtained masses were: $m_{Y_{u}}=(4.49 \pm 0.11) \mathrm{GeV}$ and $m_{Y_{s}}=(4.65 \pm 0.10) \mathrm{GeV}$. Therefore, the authors concluded that it is possible to interpret the $Y(4660)$ meson as a $[c s][\bar{c} \bar{s}]$ diquark-antidiquark state, and this is consistent with the di-pion 
invariant mass spectra shown in ref. [31] for $Y(4660)$, since there is some indication that it has a well defined intermediate state consistent with $f_{0}(980)$.

To summarize, the discovery of the $Y(4260), Y(4360)$ and $Y(4660)$ appears to represent an overpopulation of the expected charmonium $1^{--}$states. The absence of open charm production is also inconsistent with a conventional $c \bar{c}$ explanation. Possible explanations for these states include charmonium hybrid and $D_{0} \bar{D}^{*}$ or $D \bar{D}_{1}$ molecular state for $Y(4260)$, charmonium hybrid and $[c s]_{S=0}[\bar{c} \bar{s}]_{S=0}$ in a $P$-wave tetraquark state for $Y(4360)$, and a symmetrical $[c s]_{S=1}[\bar{c} \bar{s}]_{S=0}$ tetraquark state or a canonical $5{ }^{3} \mathrm{~S}_{1} c \bar{c}$ state for $Y(4660)$. The current situation regarding the $1^{--}$states produced via ISR is clearly unsettled.

\section{The $Z^{+}(4430)$ meson}

All states discussed so far are electrically neutral. The real turning point in the discussion about the structure of the new observed charmonium states was the observation by Belle Collaboration of a charged state decaying into $\psi^{\prime} \pi^{+}$, produced in $B^{+} \rightarrow K \psi^{\prime} \pi^{+}$[40]. The measured mass and width of this state is $M=(4433 \pm 5) \mathrm{MeV}, \Gamma=\left(45_{-18}^{+39}\right) \mathrm{MeV}$. There are no reports of a $Z^{+}$signal in the $J / \psi \pi^{+}$decay channel. Since the minimal quark content of this state is $c \bar{c} u \bar{d}$, this state is a prime candidate for a multiquark meson. Since $Z^{+}(4430)$ was observed in the $\psi^{\prime} \pi^{+}$channel, it is an isovector state with positive $G$-parity: $I^{G}=1^{+}$.

There are many theoretical interpretations for the $Z^{+}(4430)$ structure. Because its mass is close to the $D^{*} D_{1}$ threshold, Rosner [41] suggested it is an $S$-wave threshold effect, while others considered it to be a strong candidate for a $D^{*} D_{1}$ molecular state $[42,43,44]$. Other possible interpretations are tetraquark state [45], or a cusp in the $D^{*} D_{1}$ channel [46]. The tetraquark hypothesis implies that the $Z^{+}(4430)$ will have neutral partners decaying into $\psi^{\prime} \pi^{0} / \eta$.

Considering the $Z^{+}(4430)$ as a loosely bound $S$-wave $D^{*} D_{1}$ molecular state, the allowed angular momentum and parity are $J^{P}=0^{-}, 1^{-}, 2^{-}$, although the $2^{-}$assignment is probably suppressed in the $B^{+} \rightarrow Z^{+} K$ decay by the small phase space. Among the remaining possible $0^{-}$and $1^{-}$states, the former will be more stable as the later can also decay to $D D_{1}$ in $S$-wave. Moreover, one expects a bigger mass for the $J^{P}=1^{-}$state as compared to a $J^{P}=0^{-}$state.

In ref. [43] the QCD sum rules were used to study the $Z^{+}(4430)$ considered as a $D^{*} D_{1}$ molecular state with $I^{G} J^{P}=1^{+} 0^{-}$. The mass obtained was $M_{Z^{+}}=(4.40 \pm 0.10) \mathrm{GeV}$ in an excelent agreement with the experimental mass. To check if the $Z^{+}(4430)$ could also be described as a diquark-antidiquark state, in ref. [47] different currents were considered with $J^{P}=0^{-}$and $1^{-}$. The results obtained were: $M_{Z}\left(0^{-}\right)=(4.52 \pm 0.09) \mathrm{GeV}$ and $M_{Z}\left(1^{-}\right)=(4.84 \pm 0.14) \mathrm{GeV}$. From these results we conclude that while it is also possible to describe the $Z^{+}(4430)$ as a diquark-antidiquark state with $J^{P}=0^{-}$, the $J^{P}=1^{-}$configuration is disfavored.

Summarizing, the only open options for the $Z^{+}(4430)$ structure are tetraquark, molecule and threshold effect. It is important to mention that during this conference it was related [48] that BaBar claim no significant evidence for the existence of the $Z^{-}(4430)$ in the decay $B^{-, 0} \rightarrow$ $J / \psi\left(\psi^{\prime}\right) \pi^{-} K^{0,+}[49]$. Therefore, a confirmation of the existence of the $Z^{ \pm}(4430)$ is critical before a complete picture can be drawn. 


\section{The $Z_{1}^{+}(4050)$ and $Z_{2}^{+}(4250)$ mesons}

The $Z^{+}(4430)$ observation motivated studies of other $\bar{B}^{0} \rightarrow K^{-} \pi^{+}(c \bar{c})$ decays. In particular, the Belle Collaboration has recently reported the observation of two resonance-like structures in the $\pi^{+} \chi_{c 1}$ mass distribution [50]. The significance of each of the $\pi^{+} \chi_{c 1}$ structures exceeds $5 \sigma$ and, if they are interpreted as meson states, their minimal quark content must be $c \bar{c} u \bar{d}$. They were called $Z_{1}^{+}(4050)$ and $Z_{2}^{+}(4250)$, and their masses and widths are $M_{1}=\left(4051 \pm 14_{-41}^{+20}\right) \mathrm{MeV}$, $\Gamma_{1}=82_{-17-22}^{+21+47} \mathrm{MeV}, M_{2}=\left(4248_{-29-35}^{+44+180}\right) \mathrm{MeV}, \Gamma_{2}=177_{-39-61}^{+54+316} \mathrm{MeV}$. Since they were observed in the $\pi^{+} \chi_{c 1}$ channel, the only quantum numbers that are known about them are $I^{G}=1^{-}$.

Due to the closeness of the $Z_{1}^{+}(4050)$ and $Z_{2}^{+}(4250)$ masses to the $D^{*} \bar{D}^{*}(4020)$ and $D_{1} \bar{D}(4285)$ thresholds, these states could also be interpreted as molecular states or threshold effects. Lie et al. [51], using a meson exchange model find strong attraction for the $D^{*} \bar{D}^{*}$ system with $J^{P}=0^{+}$. They conclude that, if future experiments confirm the $Z_{1}^{+}(4050)$ existence, then it is probably a $D^{*} \bar{D}^{*}$ loosely bound molecular state. However, it is very difficult to understand a bound molecular state which mass is above the $D^{*} \bar{D}^{*}$ threshold.

In a recent work [38], the QCD sum rules formalism was used to study the $D^{*} \bar{D}^{*}$ and $D_{1} \bar{D}$ molecular states with $I^{G} J^{P}=1^{-} 0^{+}$and $1^{-} 1^{-}$respectively. The mass obtained for these molecular states are: $M_{D^{*} D^{*}}=(4.15 \pm 0.12) \mathrm{GeV}$, and $M_{D_{1} D}=(4.19 \pm 0.22) \mathrm{GeV}$. In ref. [52] it was found that the inclusion of the width, in the phenomenological side of the sum rule, increases the obtained mass for molecular states. This means that the introduction of the width in the sum rule calculation, increases the mass of the $D^{*} \bar{D}^{*}$ and $D_{1} \bar{D}$ molecules. As a result, the mass of the $D_{1} \bar{D}$ molecule will be closer to the observed $Z^{+}(4250)$ mass, and the mass of the $D^{*} \bar{D}^{*}$ molecule will be far from the $Z^{+}(4050)$ mass. Therefore, the authors of ref. [38] conclude that it is possible to describe the $Z_{2}^{+}(4250)$ resonance structure as a $D_{1} \bar{D}$ molecular state with $I^{G} J^{P}=1^{-} 1^{-}$quantum numbers, and that the $D^{*} \bar{D}^{*}$ state is probably a virtual state that is not related with the $Z_{1}^{+}(4050)$ resonance-like structure. Considering the fact that the $D^{*} D^{*}$ threshold (4020) is so close to the $Z_{1}^{+}(4050)$ mass and that the $\eta_{c}^{\prime \prime}\left(3^{1} S_{0}\right)$ mass is predicted to be around $4050 \mathrm{MeV}$ [27], it is probable that the $Z_{1}^{+}(4050)$ is only a threshold effect [27].

\section{Other multiquark states}

If the mesons $X(3872), Z^{+}(4430), Y(4260)$ and $Z_{2}^{+}(4250)$ are really molecular states, then many other molecules should exist. A systematic study of these molecular states and their experimental observation would confirm its structure and provide a new testing ground for QCD within multiquark configurations. In this context, a natural extension would be to probe the strangeness sector. In particular, in analogy with the meson $X(3872)$, a $D_{s} D^{*}$ molecule with $J^{P}=1^{+}$could be formed in the $B$ meson decay $B \rightarrow \pi X_{s} \rightarrow \pi(J / \psi K \pi)$. Since it would decay into $J / \psi K^{*} \rightarrow J / \psi K \pi$, it could be easily reconstructed.

In ref. [20] the QCD sum rules approach was used to predict the mass of the $D_{s} D^{*}$ molecular state. Such prediction is of particular importance for new upcoming experiments which can investigate with much higher precision the charmonium energy regime, like the PANDA experiment at the antiproton-proton facility at FAIR, or a possible Super-B factory experiment. Especially PANDA can do a careful scan of the various thresholds being present, in addition to precisely going through 
the exact form of the resonance curve. The obtained mass was: $M_{D_{s} D^{*}}=(3.97 \pm 0.08) \mathrm{GeV}$ very close to the $D^{*} D(3980)$ threshold, and about $100 \mathrm{MeV}$ bigger than the $X(3872)$ mass. This finding strongly suggests the possibility of the existence of a $D_{s} D^{*}$ molecular state with $J^{P}=1^{+}$.

Finally, considering that it was already observed the double-charmonium production in the reaction [53]

$$
e^{+} e^{-} \rightarrow J / \psi+X(3940)
$$

it seems that it would be possible the formation of the tetraquark $[c c][\bar{u} \bar{d}]$. Such state with quantum numbers $I=0, J=1$ and $P=+1$ which, following ref.[54], we call $T_{c c}$, is especially interesting. As already noted previously $[54,55]$, the $T_{c c}$ state cannot decay strongly or electromagnetically into two $D$ mesons in the $S$ wave due to angular momentum conservation nor in $P$ wave due to parity conservation. If its mass is below the $D D^{*}$ threshold, this decay is also forbidden, and this state would be very narrow.

The most attractive light antidiquark is expected to be the in the color triplet, flavor antisymmetric and spin 0 channel. Therefore, a constituent quark picture for $T_{c c}$ would be a light antidiquark in color triplet, flavor anti-symmetric and spin $0\left(\varepsilon_{a b c}\left[\bar{u}_{b} \gamma_{5} C \bar{d}_{c}^{T}\right]\right)$ combined with a heavy diquark of spin $1\left(\varepsilon_{a e f}\left[c_{e}^{T} C \gamma_{\mu} c_{f}\right]\right)$. Although the spin 1 configuration is repulsive, its strength is much smaller than that for the light diquark due to the heavy charm quark mass. This is why one does not expect a bound $T_{s S}$.

A QCD sum rule for such state gives [56]: $M_{T_{c c}}=(4.0 \pm 0.2) \mathrm{GeV}$ in a very good agreement with the predictions based on the one gluon exchange potential model [54], and color-magnetic model [57].

\section{Final Comments}

As a final remark, it is very important to find experimentally observable quantities which are sensitive to the quark content of the resonances. In ref. [58], Maiani et al. have shown that the nuclear modification factor, $R_{C P}$, defined as the ratio between the cross sections in central and peripheral collisions between relativistic heavy ions, can be used for this goal. They have shown that there is a large difference between the $R_{C P}$ for the $f_{0}(980)$ produced, in $A u+A u$ collisions at RHIC, when the $f_{0}(980)$ is assumed to be a four-quark state or a quark-antiquark meson. However, it will be very difficult to observe the new charmonium states discussed above in relativistic heavy ions collisions at LHC. Therefore, it is very important to find out other experimentally observable quantities which could be sensitive to the quark content of the resonances.

\section{Acknowledgements}

This work has been partly supported by FAPESP and CNPq-Brazil.

\section{References}

[1] S.-K. Choi et al. [Belle Collaboration], Phys. Rev. Lett. 91, 262001 (2003).

[2] V. M. Abazov et al. [D0 Collaboration], Phys. Rev. Lett. 93, 162002 (2004); D. Acosta et al. [CDF II Collaboration], Phys. Rev. Lett. 93, 072001 (2004); B. Aubert et al. [BaBar Collaboration], Phys. Rev. D 71, 071103 (2005). 
[3] K. Abe et al. [Belle Collaboration], hep-ex/0505037, hep-ex/0505038.

[4] B. Aubert et al. [BaBar Collaboration], Phys. Rev. D 74, 071101 (2006).

[5] D. Abulencia et al. [CDF II Collaboration], Phys. Rev. Lett. 98, 132002 (2007).

[6] T. Barnes and S. Godfrey, Phys. Rev. D 69, 054008 (2004).

[7] C. Cawfield et al. [CLEO Collaboration], Phys. Rev. Lett. 98, 092002 (2007).

[8] F.E. Close and P.R. Page, Phys. Lett. B 578, 119 (2004).

[9] E. S. Swanson, Phys. Rept. 429, 243 (2006).

[10] N.A. Tornqvist, Z. Phys. C 61, 525 (1994).

[11] G. Gokhroo et al., [Belle Collaboration], Phys. Rev. Lett. 97, 162002 (2006) [hep-ex/0606055].

[12] B. Aubert et al. [BaBar Collaboration], Phys. Rev. D 77, 011102 (2008) [arXiv:0708.1565].

[13] E. Braaten, arXiv:0808.2948.

[14] I. Adashi et al. [Belle Collaboration], arXiv:0810.0358.

[15] L. Maiani, V. Riquer, F. Piccinini and A.D. Polosa, Phy. Rev. D71, 014028 (2005).

[16] A.D. Polosa, arXiv:hep-ph/0609137.

[17] K. Abe et al. [Belle Collaboration], Bele-CONF-0711.

[18] B. Aubert et al. [BaBar Collaboration], arXiv:0803.2838.

[19] R.D. Matheus, S. Narison, M. Nielsen and J.-M. Richard, Phys. Rev. D75, 014005 (2007).

[20] S.H. Lee, M. Nielsen and U. Wiedner, arXiv:0803.1168.

[21] B. Aubert et al. [BaBar Collaboration], Phys. Rev. Lett. 95, 142001 (2005).

[22] Q. He et al. [CLEO Collaboration], Phys. Rev. D 74, 091104(R) (2006); C.Z. Yuan et al. [Belle Collaboration], Phys. Rev. Lett. 99, 182004 (2007).

[23] B. Aubert et al. [BaBar Collaboration], arXiv:0808.1543.

[24] B. Aubert et al. [BaBar Collaboration], Phys. Rev. Lett. 98, 212001 (2007).

[25] X.L. Wang et al. [Belle Collaboration], Phys. Rev. Lett. 99, 142002 (2007).

[26] S.-L. Zhu, arXiv:0707.2623 [hep-ph]; hep-ph/0703225; K.K. Seth, arXiv:0712.0340.

[27] S. Godfrey and S.L. Olsen, arXiv:0801.3867.

[28] K.J. Juge, J. Kuti and C.J. Morningstar, Phys. Rev. Lett. 82, 4400 (1999).

[29] T. Barnes, F.E. Close and E.S. Swanson, Phys. Rev. D52, 5242 (1995); F.E. Close and P.R. Page, Phys. Lett. B628, 215 (2005).

[30] G.S. Bali, Eur. Phys. J. A19, 1 (2004).

[31] R. Faccini, arXiv:0801.2679.

[32] F.-K. Guo, C. Hanhart and U.-G. Meissner, arXiv:0803.1392.

[33] C.F. Qiao, arXiv:0709.4066 [hep-ph].

[34] G.-J. Ding, J.-J. Zhu and M.-L. Yan, arXiv:0708.3712. 
[35] L. Maiani, V. Riquer, F. Piccinini and A.D. Polosa, Phy. Rev. D72, 031502 (2005).

[36] D. Ebert, R.N. Faustov, V.O. Galkin, arXiv:0808.3912.

[37] R.M. Albuquerque and M. Nielsen, arXiv:0804.4817.

[38] S.H. Lee, K. Morita, M. Nielsen, arXiv:0808.0690.

[39] G.-J. Ding, arXiv:0809.4818.

[40] K. Abe et al. [Belle Collaboration], Phys. Rev. Lett. 100, 142001 (2008).

[41] J.L. Rosner, arXiv:0708.3496.

[42] C. Meng, K.-T. Chao, arXiv:0708.4222.

[43] S.H. Lee, A. Mihara, F.S. Navarra and M. Nielsen, Phys. Lett. B661, 28 (2008).

[44] X. Liu, Y.R. Liu, W.Z. Deng, S.-L. Zhu, arXiv:0711.0494.

[45] L. Maiani, A.D. Polosa, V. Riquer, arXiv:0708.3997.

[46] D.V. Bugg, arXiv:0709.1254.

[47] M.E. Bracco, S.H. Lee, M. Nielsen, R. Rodrigues da Silva, arXiv:0807.3275.

[48] V. Balagura, at these proccendings.

[49] A.G. Mokhtar, arXiv:0810.1073.

[50] R. Mizuk et al. [Belle Collaboration], arXiv:0806.4098.

[51] X. Liu, Z.-G. Luo, Y.-R. Liu, S.-L. Zhu, arXiv:0808.0073.

[52] S.H. Lee, K. Morita and M. Nielsen, arXiv:0808.3168.

[53] K. Abe et al. [Belle Collaboration], Phys. Rev. Lett. 98, 082001 (2007).

[54] D. Janc and M. Rosina, Few Body Sust. 35, 175 (2004).

[55] S. Zouzou, B. Silvestre-Brac, C. Gignoux and J.M. Richard, Z. Phys. C30, 457 (1986).

[56] F.S. Navarra, M. Nielsen and S.H. Lee, Phys. Lett. B649, 166 (2007).

[57] Y. Cui, X.-L. Chen, W.-Z. Deng and S.-L. Zhu, High Energy Phys. Nucl. Phys. 31, 7 (2007).

[58] L. Maiani, A.D. Polosa, V. Riquer, C.A. Salgado, Phys. Lett. B645, 138 (2007). 